\title{
Unstable periodic orbits and attractor of the barotropic ocean model
}

\author{
E. Kazantsev \\ INRIA-Lorraine, projet NUMATH. Nancy, France
}

Received: 21 February 1998 - Revised: 08 January 1999 - Accepted: 12 February 1999

\begin{abstract}
A numerical method for detection of un ${ }^{-}$ stable periodic orbits on attractors of nonlinear models is proposed. The method requires similar techniques to data assimilation. This fact facilitates its implcmentation for geophysical models. This method was used to find numerically several low-period orbits for the barotropic ocean model in a square. Some numerical particularitics of application of this method are discussed.

Knowledge of periodic orbits of the model helps to explain some of these featurcs like bimodality of probability density functions (PDF) of principal parameters. These PDFs have been reconstructed as weighted averages of periodic orbits with weights proportional to the period of the orbit and inversely proportional to the sum of positive Lyapunov exponents.

The fraction of time spent in the vicinity of each periodic orbit has been compared with its instability characteristics. The relationship between these values shows the $93 \%$ correlation. The attractor dimension of the model has also been approximated as a weighted average of local attractor dimensions in vicinities of periodic orbits.
\end{abstract}

\section{Introduction.}

Numerous recent studies have been focussed on the properties of chaotic solutions generated by nonlinear systems. One of the major fields, where this kind of solution is extremely important, is atmospheric and oceanic dynamics. The importance is based on the necessity to deliver weather forecasts and the limited time of deterministic forecasting of chaotic systems. The success of

\footnotetext{
Correspondence to: E. Kazantsev, INRIA-Lorraine, projet NUMATH, 615, rue du Jardin Botanique, BP101, 54602, Villers-lès-Nancy, Cedex, France.

F-mail: kazan@loria.fr
}

long-range forecasting depends on the understanding of the sources and nature of the variability of the system beyond the timescale of deterministic prediction.

Low frequency variability of nonlinear dynamics of the atmosphere has long been discussed. After the discovery of deterministic chaos (Lorcnz, 1963) the interpretation of the atmospheric and oceanic circulations, their variability and predictability, has been developed using tools of the dynamical systems theory.

One of the approaches to apply the dynamical system theory to atmospheric and oceanic dynamics consists of the extraction of local characteristics of the strangc attractor. It is well known that the behaviour of a solntion can differ a lot on different parts of the attractor. The attractor inhomogeneity leads to the variability of the predictability time scale in the phase space. This variability can result, for example, from the existence of multiple unstable stationary points on the attractor. The predictability time of the system can be influenced in vicinities of these points and their stable and unstable manifolds. This idea has been quantified in various ways by using the information about local characteristics of the phase space.

The study of this kind of inhomogenesty for almospheric and oceanic dynamics was started by the work of Charney and De Vore (1979). They showed that a system of atmospheric flows in a rotating channel with a spatially inhomogeneous forcing can have several unstable equilibria.

The theory of multiple equilibria (Charney and Do Vore, 1979) of the atmospheric circulation developed in order to explain and classify quasi-stationary atmospherio régimes is a good example of the application of dynamical systems theory to the climate. This apmorich allowed quantification of many properties of multiple weather régimes, that have been addressed in munerous analytical and observational studies during the past fifty years.

The earliest notions of their classifications relate to 
high and low circulation indices (Rossby, 1939), zonal and meridional circulation indices (Blinova, 1943), blocked and zonal flows (Berggren et al., 1949), (Rex, 1950). These empirical classifications have been revisited and their properties have been explained based on the hypothesis that when a system passes a state close to one of multiple equilibria, quasi-stationary atmospheric régimc results.

Each hyperbolically unstable equilibrium forms a region in the phase space where the system trajectories slow down before being ejected along an unstable manifold. The validity of this hypothesis has been confirmed by comparison of the results of cluster analysis of the solution of the barotropic model with its equilibria (Mo and Ghil, 1988). The analysis shows closeness of the riuster centroids with the equilibria.

Using a truncated barotropic model of the atmosphere, Legras and Ghil (1985) demonstrated that recurrent quasistationary states occur in vicinities of unstable stationary points in the phase space of the model. The lifetime of this régime depends on a particular trajectory in the phase space (Mo and Ghil, 1987). However, the mean lifetime of such a régime related to the stability characteristics of the adjacent stationary point (Dymnikov et al., 1990). This fact allows us to obtain a priori estimates of the régime's lifctime. The climatic average of the barotropic model solution has been analysed by Dymnikov and Kazantsev (1993). It is shown that this average can be approximated by the set of equilibria with a rather good accuracy.

Essential results have recently been obtained from theoretical investigations of the phase space structures. Theorcms for the cxistence and uniqueness of a solution and existence of the finite-dimensional attractor for geophysical models have been proved by Temam (1988), Dymnikov and Filatov (1990), Bernier (1994). Analysis of the number of equilibria in the phase space of the model has been performed by Filatov (1992). Mathenuatical analysis of climatic processes can be found in Dymnikov and Filatov (1996). These works provide a good theoretical basis for the forthcoming studies.

However, the applications of this theory to the analysis of climatic models possesses one principal "shortcoming". Despite the fact that there exist multiple equilibria, their vicinities cover a very small part of the attractor only. Thus, the model solution sperds much time out of these vicinities where its behaviour can not be explained by this theory.

So far, the analysis of equilibrium points of chaotic models has been fruitful; one can try to study another kind of "particular solution" of a nonlinear system. This kind of solution involves the periodic: orbits (or linnit cycles) which can also exist on the attractor of the system. As well as equilibria, discussed above, they can be stable and unstable. However, in practice, only unstable equilibria and unstable periodic orbits require special attention in the studies of a chaotic system, because any stable solution usually forms a regular attractor with no chaos.

Similarly to quasi-stationary régimes, which are cxplained by the motion near unstable equilibrium, we can speak of quasi-oscillatory régimes, or intermittent appearances of oscillatory modes, which may be explained by the motion in proximity to an unstable linit cycle.

The periodic orbits for a geophysical model have attracted some interest recently ((Itoh and Kimoto, 1996), (Jiang et al., 1995), (Jiang and Ghil, 1997), (Dymnikov ct al., 1998)). However, all these papers address either stable periodic orbits, or periodic orbits near the Hopf bifurcation point where weakly nonlinear thcory can be. applied.

The principal difference between stationary solutions and periodic orbits is that the number of equilibria is usually finite for a geophysical model. Moreover, it is always finite for a finite dimensional approximation of a model, which includes a polynomial nonlinearity only. If the nonlinearity is quadratic, the number of stationary points is bounded by $2^{N}$, where $N$ is number of degrees of freedom of the discretisation. So one can hope to find all equilibria of the model. Howcver, the number of periodic orbits is usually infinite even for a discretised model. To say more, one of definitions of the chaotic system (Devancy, 1987) defines the set $V$, where the behaviour of the system is chaotic, as the sct on which periodic orbits are dense. Following this definition, if wo speak of a chaotic system, its periodic orbits are dense in some $V$, hence they are of infinite number. This is the casc, for example, of the Lorenz system (Lorenz, 1963), which is composed of three ordinary differential equations only.

Despite the impossibility to find all the periodic orbits, we can find and analyse some of them, with lowest. periods. The situation is similar in some sense to the equilibria analysis in the case when we do not know whether we found all of them or not. In this case, we have to analyse only the equilibria we have found.

However, only a limited number of low-period orbits may be sufficient for some purposes. This point of view is argued by Hunt and Ott (1996a) and by Hunt and Ott (1996b). This conclusion does not hold generally and some applications may require long-period orbits as well (Zoldi and Greenside, 1997b).

The instability of periodic orbits of chaotic: systems makes the problem of their numerical search more complicated. They can not be found by a simple stabilisation method generally uscdto find stable orbits. Therefore, some special algorithm must be applied for this purpose. In this paper, we use the algorithm developed and tested by Kazantsev (1998) to find several low-period orbits of the barotropic ocean model.

There is another difference between periodic: orbits and stationary points on the attractor of a nonlinear model. This difference consists of the lack of theoretical results available for periodic orbits. Even the existence? 
of periodic orbits is doubtful for a PDE system like the barotropic model. So we study periodic orbits of the finite dimensional ODE system, obtained after discretisation, and we can state nothing about orbits of the original system.

So, periodic orbits of the discretised system are discussed only in this paper. In the first section of the paper, the model equations are presented and the method of unstable periodic orbits search is discussed. The second section is devoted to the analysis of their properties related to the predictability and attractor of the model.

\section{Barotropic ocean model in a square}

\subsection{Model and its parameters.}

We consider barotropic ocean dynamics, i.c. all the thermodynamic effects are neglected and the vertical structure of the occan is supposed to be uniform. The equation of dynamics of the wind-driven ocean is written for the barotropic vorticity $\omega$

$\frac{\partial \omega}{\partial t}+\mathcal{J}(\psi, \omega+\ell)=\mu \Delta \omega-\sigma \omega+f, \quad \omega=\Delta \psi$

We assume the $\beta$-plane approximation for the Coriolis parameter $\ell$, which represents the effect of the Earth rotation in this equation, i.c. we suppose that this parameter is lincar in the $y$ coordinate: $\ell=\ell_{0}+\beta y$, where $\ell_{0}$ is the value of the Coriolis parameter at the mid-latitude of the basin. We use very simple basin geometry represented by a squarc box of side length $L=4000 \mathrm{~km}$. We suppose that this basin is located in the middle of the North Atlantic, so we take the value of the Coriolis parameter in the middle of the basin to be equal to $\ell_{0}=9.3 \times 10^{-5} s^{-1}$, and its meridional gradient $\beta=2 \times 10^{-11} m^{-1} s^{-1}$.

The source of cnergy in this equation is presented by the atmospheric wind stress applied to the surface. In this paper, we take a steady zonal wind with a now classical two gyre antisymmetric pattern. This is scen as a schematic pattern for the mean curl of the wind stress over the North Atlanitic ocean in middle latitudes. Its magnitude is equal to

$f=-\frac{2 \pi \tau_{0}}{\rho H L} \sin \frac{2 \pi y}{L}$,

where $\tau_{0}=1.1$ dyne $\mathrm{cm}^{-2}$ is the wind tension on the surface, $\rho=1000 \mathrm{~kg} \mathrm{~m}^{-3}$ is the density of water. The depth of the active wind driven current $H$ has been chosen as $500 \mathrm{~m}$. The dissipation in the equation (1) is composed by the harmonic lateral friction $\mu \Delta \omega$ and the bottom drag parametrised by $\sigma \omega$. Values of friction coefficients used in this paper are $\mu=1250 \mathrm{~m}^{2} \mathrm{~s}^{-1}$ and $\sigma=5 \times 10^{-8} s^{-1}$.

The equation (1) is subjected to impermeability and frec-slip boundary conditions

$\varphi_{1, \Omega}=0,\left.\quad \omega\right|_{a \Omega}=0$.
We do not intend to reproduce actual oceanographic: data, which would be hopeless in frames of a barotropic model. The model is used in this paper just as a simple well-studied geophysical model to test the method of periodic orbit search and to test the possibility to explain some properties of a multi-dimensional chaotic system by means of its unstable periodic orbits.

In order to look for a weak solution of the problem (1), (3), we perform its variational formulation:

$$
\begin{aligned}
<\frac{\partial \omega}{\partial t}, \varphi>+\langle\mathcal{J}(\psi, \omega+\beta y), \varphi> & = \\
=-\mu<\nabla \omega, \nabla \varphi>-\sigma<\omega, \varphi> & +\langle f, \varphi> \\
\langle\omega, \varphi> & =-\langle\nabla \psi, \nabla \varphi>.
\end{aligned}
$$

for any function $\varphi(x, y) \in H_{0}^{1}(\Omega)$. Here, $H_{0}^{1}(\Omega)$ denotes the linear space of functions, the square of which is integrable as well as the square of their first derivatives. In addition, functions in this space must vanist on the boundary of the domain. Brackets $\langle\ldots\rangle$ demote the $L_{2}$ scalar product:

$<\psi, \varphi>=\iint_{\Omega} \psi \varphi d x d y$

\subsection{Discretisation.}

The variational formulation (4), (5) of the problem (1) allows to look for a solution by the finite element method (FEM). The feasibility and utility of FEMs for modclling ocean dynamics was first addressed by Fix (1975). He stated that using FEMs brought numerous advantages of modelling like precision, natural conservation of model invariants, flexibility of discretisation of complex domains, etc. These advantages remain even when irregular discretisation of the domain is performed.

So far, the solution produced by the barotropic model of the North Atlantic typically includes a western boundary layer with intense velocity gradients; the advantage of refining the triangulation along the western boundary of the domain is rather clear. This helps to keep the quality of explicit eddy resolution by the model while working with a lower number of grid nodes. The comparison of finite elcment (FE) and finite difference (FD) models performed in Le Provost et al. (1994) revealed that the difference arising between simulations by $\mathrm{FE}$ and FD techniques can be regarded as insignificant when the number of FE nodes is about 6 times lower than the number of FD ones.

In spite of the fact that the number of operations per time step and grid node is much higher for the FE model, the possibility of reducing the number of grid points considerably diminishes the computational cost of a model run. The possibility of a better precision working with fewer grid points is very valuable in this work due to the high number of operations per point. Another atvantage of using the FE model is the simplicity of the adjoint model formulation. This aspect will be discussed below. 


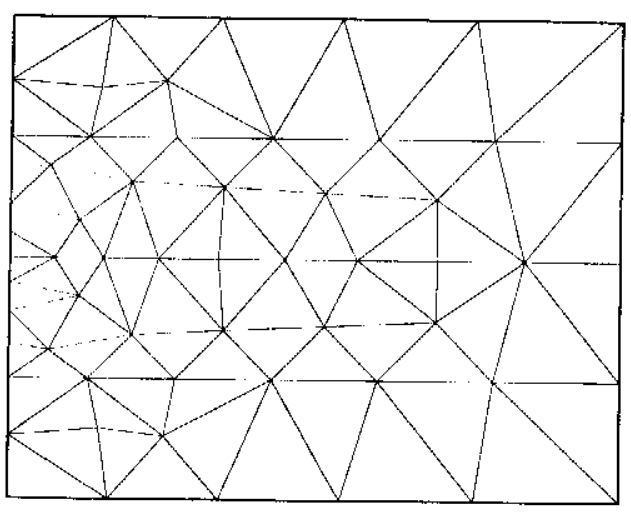

Fig. 1. Triangulation of a unit square. The integration points set is the union of vertices and centers of edges of triangles.

The package MODULEF (Bernadou, 1988) has been used to perform a triangulation of a domain. This package produces quasi-regular triangulation of the domain, based upon the prescribed grid nodes on its boundary. We require the refining of the triangulation near the western boundary and especially in the middle of the domain, where velocity gradients are extremely sharps.

The domain $\Omega$ is covered by a set of non-intersecting triangles. The set of integration points is defined as the union of vertices and centers of edges of triangles. Finite elements of type $P_{2}$ are used here, i.e. the polynomials of the second degree $p_{i}(x, y)=a_{i} x^{2}+b_{i} x y+c_{i} y^{2}+d_{i} x+$ $e_{i} y+f_{i}$. The $i$ th finite element is taken to be equal to 1 at the i-th integration point and zero at all other points.

The grid used in this paper is presented in fig.1. This triangulation is composed of 92 triangles. The integration points set, being a union of vertices and mi-edges of triangles, counts 211 nodes. The resolution of the grid varies between $1 / 40$ of the side length (about $100 \mathrm{~km}$ ) near the western boundary and $1 / 7$ of the side length (about $550 \mathrm{~km}$ ) ncar the eastern one.

According to the Dirichlet boundary conditions (3), we consider internal points of the domain only: $\left(x_{i}, y_{i}\right) \in$ $\Omega \backslash \partial \Omega$ for $i=1, \ldots, N$, so the functions $\psi, \omega$ arc presented as linear combinations

$\psi(x, y, t)=\sum_{i=1}^{N} \psi_{i}(t) p_{i}(x, y)$,

$\omega(x, y, t)=\sum_{i=1}^{N} \omega_{i}(t) p_{i}(x, y)$.

To simplify notations, we define matrices of mass and rigidity as

$$
\begin{array}{r}
\mathcal{M}_{i, j}=\left\langle p_{i}, p_{j}>, \mathcal{C}_{i, j}=\left\langle\nabla p_{i}, \nabla p_{j}\right\rangle,\right. \\
\left\{\begin{array}{l}
i=1, \ldots, N \\
j=1, \ldots, N
\end{array}\right.
\end{array}
$$

Using these expressions, we can write the discretised system (4):

$$
\begin{gathered}
\mathcal{M} \frac{\partial \omega}{\partial t}+\mathcal{J}(\psi, \omega+\beta y)=-\mu \mathcal{C} \omega-\sigma \mathcal{M} \omega+\mathcal{M} f \\
\mathcal{M} \omega=-\mathcal{C} \psi \\
\mathcal{J}(\psi, \omega+\beta y)_{j}=\sum_{i} \sum_{m} \psi_{m}\left(\omega_{i}+\beta y_{i}\right)<\mathcal{J}\left(p_{m}, p_{i}\right), p_{j}>
\end{gathered}
$$

This system has been integrated in time as follows

$$
\begin{aligned}
& \mathcal{M} \frac{\omega^{n+1}-\omega^{n-1}}{2 \tau}+\mathcal{J}\left(\psi^{n}, \omega^{n}+\beta y\right)= \\
& =-\mu \mathcal{C} \frac{\omega^{n+1}+\omega^{n-1}}{2}-\sigma \mathcal{M} \frac{\omega^{n+1}+\omega^{n-1}}{2}+\mathcal{M f}
\end{aligned}
$$

The first step is performed by the Euler scheme for the nonlinear advection term:

$$
\begin{aligned}
\mathcal{M} \frac{\omega^{2}-\omega^{1}}{\tau} & +\mathcal{J}\left(\psi^{1}, \omega^{1}+\beta y\right)= \\
& =-\mu \mathcal{C} \frac{\omega^{2}+\omega^{1}}{2}-\sigma \mathcal{M} \frac{\omega^{2}+\omega^{1}}{2}+\mathcal{M f}
\end{aligned}
$$

\section{Detection of unstable periodic orbits.}

The problem of numerical search for unstable pcriodic orbits is not new. Sparrow (1982) has proposed to use the Newton method to locate unstable periodic orbits on the attractor of the Lorenz model.

However, one has to choose initial conditions in a rather close vicinity of a periodic orbit, otherwise the method diverges. To simplify the choice of the initial point, a dumped-Newton method has been proposed to use by Zoldi and Greenside (1997a). This method differs from the classical Newton method by the fraction $\alpha \leq 1$ of a Newton correction $\delta x$, which is added only to update the unknowns, $x \rightarrow x+\alpha \delta x$. This method is found to be more efficient to find periodic orbits due to the less restrictive choice of the initial gucss.

However, realisation of this method rcquires $O\left(N^{3}\right)$ operations per iteration due to the necessity of calculating the matrix of the Newton process and solving the systern of equations with this matrix. This fact limits its use to low-dimensional systerns only. In fact, methods of this kind work well for the Lorenz system and even for higher dimensional systems; lowever, the number of variables must not exceed 100 .

Several methods based on the stabilisation of unstabl. periodic orbits have also been proposed. Barreto (n al (1997) have discussed the possibility of finding a "window" in the parameter range of the model, where one of its periodic orbits becomes stable. However, this kind of "window" depends on the number of positive Lyapunov exponents on the attractor and, if this number is sufficiently large, the search of such a "window" may become difficult or impossible. Moreover, it may also be difficult to know whether pcriodic orbits are subject. to 
smooth variations only or if bifurcations may occur under parameter variations. If secondary Hopf bifurcation can occur, non-existing orbits can be found.

In Schmelcher and Diakonos (1997), a method of stabilisation of orbits is based on a universal set of linear transformations, namely special reflections and rotidtions in spacc. However, the application of this method to a high dimensional system may hardly be efficient due to the very large number of possible transformations.

To find periodic orbits of the barotropic occan model we note the great progress achicved in variational data assimilation techniques. Data assimilation procedures based on a functional minimisation have been developed for many models. Moreover, this minimisation uses gradient-type methods, which require as many operations per iteration as the model does, i.c. $O\left(N_{t} \times N_{x}^{3 / 2}\right)$ or even $O\left(N_{t} \times N_{x} \ln N_{x}\right)$. If we formulate the problem of periodic orbit search as a functional minimisation problem, we can apply a technique similar to variational data assimilation.

\subsection{Variational problem}

Suppose the initial conditions for the model (1) are written as $\omega(x, y, 0)=\xi(x, y)$. Let the barotropic flow be written as a function of initial condition and time

$\omega(x, y, t)=S(\xi, t)$,

that is, $S$ is the semigroup gencrated by the system (1).

Let us consider the functional

$J(\xi, T)=\frac{\|\omega(x, y, T)-\omega(x, y, 0)\|^{2}}{2}=\frac{\|S(\xi, T)-\xi\|^{2}}{2}$

where the norm $\|\eta\|$ is associated with a scalar product in the finite dimensional space

$\ll \psi, \varphi\rangle=\sum_{i}(\mathcal{H} \psi)_{i} \varphi_{i}=(\mathcal{H} \psi, \varphi)$

and where $\mathcal{H}$ is some symmetric positive definite matrix. The choice of this matrix will be discussed later. We can just note now that if $\mathcal{H}$ is equal to the mass matrix, the scalar product (12) becomes a finite dimensional approximation of the $L_{2}$ scalar product (5). If $\mathcal{H}=I$, we shall work with a simple Euclidian product in $R^{N}$ space (from here and below $I$ will denote the identity matrix).

One can easily see that for any periodic solution of pcriod $T$ the value of this functional $J(\xi, T)$ is equal to 0 for all points $\xi$ of the periodic solution. And if $J(\xi, T)=0$ for some $\xi$ and $T$, the orbit originating at $\xi$ is a periodic one with period $T$. Thus, the purpose is to find points where $J(\xi, T)$ vanishes. It should be noted that the value of the period $T$ is unknown, hence one must work in the $N+1$ variables space composed of $N$ components of the vector $\xi$ and the period $T$. So fir this functional is always non-negative; we can look for its minima instead of looking for its zeroes.
To minimise $J$ we calculate first its gradient at a givon point $\xi$ and given $T$ in the $N+1$ variables space. Thr variation of $J(\xi, T)$ under variations of the initial peint $\xi$ can be writtcn

$$
\begin{aligned}
& \delta_{\xi} J(\xi, T)=\delta_{\xi} \frac{\|S(\xi, T)-\xi\|^{2}}{2}= \\
& =\ll S(\xi, T)-\xi,\left[\frac{\partial S(\xi, T)}{\partial \xi}\right] \delta \xi-\delta \xi+o(\delta \xi) \gg,
\end{aligned}
$$

where $\frac{\partial S(\xi, T)}{\partial \xi}$ is the matrix of the tangent linear model integrated for time $T$. This model can be obtained easily as the linearisation of the (1) around its trajectory $S(\xi, t)$ for $t \in[0, T]$ :

$$
\frac{\partial \omega}{\partial t}+\mathcal{J}\left(\Delta^{-1} S(\xi, t), \omega\right)+\mathcal{J}(\psi, S(\xi, t)+\ell)=\mu \Delta \omega-\sigma \omega .
$$

This model has been discretised in the same way as the model (1). Finite elements were used to perform spatial discretisation and equation (8) was applied for time stepping.

$$
\begin{array}{r}
\mathcal{M} \frac{\omega^{n+1}-\omega^{n-1}}{2 \tau}+\mathcal{J}\left(\Delta^{-1} S\left(\xi, t^{n}\right), \omega^{n}\right)+ \\
+\mathcal{J}\left(\psi^{n}, S\left(\xi, t^{n}\right)+\beta y\right)= \\
=-\mu \mathcal{C} \frac{\omega^{n+1}+\omega^{n-1}}{2}-\sigma \mathcal{M} \frac{\omega^{n+1}+\omega^{n-1}}{2}
\end{array}
$$

So far, the integration of the model requires two former steps to calculate the next one; the state vector has to be $2 N$ dimensional and be composed of vorticity vectors on the $n$th and $n-1$ time steps. Using this notation, this system can be rewritten in a compact matricial form.

$$
\begin{aligned}
\left(\begin{array}{c}
\omega^{n+1} \\
\omega^{n}
\end{array}\right) & =\left(\begin{array}{cc}
2 \tau E_{+}^{-1} \mathcal{I}\left(S\left(\xi, t^{n}\right)\right) & E_{+}^{-1} E_{-} \\
I & 0
\end{array}\right) \times \\
& \times\left(\begin{array}{c}
\omega^{n} \\
\omega^{n-1}
\end{array}\right)
\end{aligned}
$$

whore

$$
\begin{gathered}
E_{+}=\mathcal{M}+r \sigma \mathcal{M}+\tau \mu \mathcal{C}, \quad E_{-}=\mathcal{M}-\tau \sigma \mathcal{M}-\tau \mu \mathcal{C} \\
\begin{aligned}
\mathcal{I}\left(S\left(\xi, t^{n}\right)\right) \varphi & =-\mathcal{J}\left(\Delta^{-1} S\left(\xi, t^{n}\right), \varphi\right)- \\
& -\mathcal{J}\left(\Delta^{-1} \varphi, S\left(\xi, t^{n}\right)+\beta y\right)
\end{aligned}
\end{gathered}
$$

As before, the first step is performed by the Euler scheme for the nonlinear advection term, so the matrix of the first step is $2 N \times N$ rectangular

$$
\left(\begin{array}{c}
\omega^{2} \\
\omega^{1}
\end{array}\right)=\left(\begin{array}{c}
\mathcal{K} \\
I
\end{array}\right) \omega^{1}
$$

where

$$
\begin{aligned}
\mathcal{K} & =\left(\mathcal{M}+\frac{\mu \tau}{2} \mathcal{C}+\frac{\sigma \tau}{2} \mathcal{M}\right)^{-1} \times \\
& \times\left(\tau \mathcal{I}\left(S\left(\xi, t^{1}\right)\right)+\mathcal{M}-\frac{\mu \tau}{2} C-\frac{\sigma \tau}{2} \mathcal{M}\right)
\end{aligned}
$$


Hence, the matrix $\frac{\partial S(\xi, T)}{\partial \xi}$ can be obtained as the result of the tangent linear model integration from $t=0$ to $t=T$, or as the product of time-step matrices (18), (16).

To develop the adjoint of this tangent linear model, we calculate first the adjoint to the Jacobian terms of the (14):

$$
\begin{aligned}
<\mathcal{J}(\bar{\psi}, \omega), \omega^{*}> & =\left\langle-\mathcal{J}\left(\bar{\psi}, \omega^{*}\right), \omega\right\rangle, \\
<\mathcal{J}(\psi, \bar{\omega}+\ell), \omega^{*}> & =-\left\langle\mathcal{J}\left(\omega^{*}, \bar{\omega}+\ell\right), \Delta^{-1} \omega>=\right. \\
& =<\Delta^{-1} \mathcal{J}\left(\bar{\omega}+\ell, \omega^{*}\right), \omega>
\end{aligned}
$$

Therefore, the adjoint of the linearised Jacobian is

$$
\begin{aligned}
\mathcal{I}^{*}(S(\xi, t)) \omega^{*} & =\mathcal{J}\left(\Delta^{-1} S(\xi, t), \omega^{*}\right)- \\
& -\Delta^{-1} \mathcal{J}\left(S(\xi, t)+\ell, \omega^{*}\right)
\end{aligned}
$$

So far, the operators $E_{+}, E_{-}$are self-adjoint as well as their inverses, the adjoint model can be written

$$
\left(\begin{array}{c}
\omega_{n-1}^{*} \\
\omega_{n}^{*}
\end{array}\right)=\left(\begin{array}{cc}
2 \tau \mathcal{I}^{*}\left(S\left(\xi, t^{n}\right)\right) E_{+}^{-1} & I \\
E_{-} E_{+}^{-1} & 0
\end{array}\right)\left(\begin{array}{c}
\omega_{n}^{*} \\
\omega_{n+1}^{*}
\end{array}\right)
$$

It should be noted here that not only $\omega_{n-1}^{*}$ is calculated at each step, but also $\omega_{n}^{*}$ is modified. The modificd value is used at the next step to calculate $\omega_{n-2}^{*}$.

The last step of the adjoint model integration is the adjoint to the first step of the tangent model (18):

$$
\begin{aligned}
\omega_{1}^{*} & =\left(\mathcal{M}+\frac{\mu \tau}{2} \mathcal{C}+\frac{\sigma \tau}{2} \mathcal{M}\right)^{-1} \times \\
& \times\left(\tau \mathcal{I}\left(S\left(\xi, t^{1}\right)\right)+\mathcal{M}-\frac{\mu \tau}{2} \mathcal{C}-\frac{\sigma \tau}{2} \mathcal{M}\right) \omega_{2}^{*}+\omega_{3}^{*}
\end{aligned}
$$

Using the notation of the adjoint Inodel, the expression (13) can be rewritten as

$$
\begin{gathered}
\delta_{\xi} J(\xi, T)=\ll S(\xi, T)-\xi,\left[\frac{\partial S(\xi, T)}{\partial \xi}\right] \delta \xi-\delta \xi \gg+o(\delta \xi) \\
=\left(\mathcal{H}(S(\xi, T)-\xi),\left[\frac{\partial S(\xi, T)}{\partial \xi}\right] \delta \xi-\delta \xi\right)+o(\delta \xi)= \\
=\left(\left(\left[\frac{\partial S(\xi, T)}{\partial \xi}\right]^{*}-I\right) \mathcal{H}(S(\xi, T)-\xi), \delta \xi\right)+o(\delta \xi(24)
\end{gathered}
$$

where $\left[\frac{\partial S(\xi, T)}{\partial \xi}\right]^{*}$ is the matrix of the adjoint model integrated from $t=T$ to $t=0$ following (22) and (23). Initial conditions for the integration of the adjoint model can be written as $\omega_{t=T}^{*}=\mathcal{H}(S(\xi, T)-\xi)$.

The gradient of the functional is the linear part of its variation when variations of the argument are proportional to unitary vectors $\delta \xi=\varepsilon e_{i}$. So the first $N$ components of the gradient can be obtained from (24)

$\nabla_{\xi} J(\xi, T)=\left(\left[\frac{\partial S(\xi, T)}{\partial \xi}\right]^{*}-I\right) \mathcal{H}(S(\xi, T)-\xi)$

and the last component is the partial derivative with respect to $T$ :

$\frac{\partial . J(\xi, T)}{\partial T}=\ll \frac{\partial S(\xi, T)}{\partial T}, S(\xi, T)-\xi \gg$.
We recall that it follows, from (10) and (1), that

$$
\begin{aligned}
\frac{\partial S(\xi, T)}{\partial T} & =\left.\frac{\partial \omega(x, y, t)}{\partial t}\right|_{i=T}= \\
& =(-\mathcal{J}(\psi, \omega+\ell)+\mu \Delta \omega-\sigma \omega+f)_{t=T}
\end{aligned}
$$

Hence, the last component of the gradient is simply the scalar product of the right-hand side of equation (1) calculated at the final point of the integration, and the difference between the final point and initial point.

Thus the gradient at any given point $\xi$ and for a givon $T$ can be calculated by the dircct model (8) run for time $T$, scalar product (26) calculation and the adjoint model (22) run. It should be noted here that one has to keep the entire orbit $S(\xi, t), t \in[0, T]$ for further integration of the adjoint model.

The gradient of $J\left(\xi_{n}, T_{n}\right)$ allows us to perform the iterative descent from the point $\xi_{n}, T_{n}$ to the point $\xi_{n+1}$, $T_{n+1}$. The minimisation procedure nsed here was developed by Gilbert and Lemarechal (1989). The procedure uses the limited momory quasi-Newton method.

\subsection{Numerical realisation.}

The initial point of iterations $\xi_{0}, T_{0}$ can be chosen as an arbitrary point on the attractor of the model. For example, one can integrate the model for an arbitrary time and take the result of the integration as the starting point for iterations. The minimisator prohibits the process divergence, so there exist three possibilitic.i only of the process development:

- the process converges to a minimum with some nonzero $J$,

- the process converges to a solution already found,

the process converges to a new solution.

Only the third possibility is considered to be useful, the first two are ignored. 'Typicat realisation of the first possibility is cansed by existence of an invariant torus on the attractor. In this (ase, the procedure converges w) the "most periodie orbit" on the torus realising the lemzero minimum. So far, we do not intend to study inwariant tori in this paper, ail non-zero minima are skippext. It should be noted that stationary points could also bo found by this method because they are a particular case of a periodic orbit with an arbitrary period.

To distinguish the new solution to the procedure from previously found orbits one has to calculate phase-spatial distance betwcen them. This is necessary because any point on the orbit can be found as its initial point. Worcover, any multiple of $T$ can be found as the period of the orbit. Obviously, any miltiply repeated orbit should be abridged to one cycle. The precision required to stop) iterations is $J<10^{-20}$. To achieve this precision alont. 1000-1500 itcrations are "1sulally required. 
One particularity of this task is the necessity to perform the integration for the exact given value of $T$. Also, the value of $T$ may vary during the descent procedure. To perform numerical run one can choose some fixed number of time steps $N_{t}$ and calculate the time step length $\tau$ as $\tau=T / N_{t}$. However, this requires $N_{t}$ to be sufficiently large that even for largest possible $T$ the value of $\tau$ never exceods the stability limit of schemes (8) and (22). This can decrease the efficiency of the method due to the need to integrate low-period orbits with a very small $\tau$. To avoid this lost of efficiency, we can choose some reasonable value of the time step, which ensures the stability of schemes and allow $N_{t}$ to vary.

To ensure the stability of the model one has to perform about 10 steps per day. The number of steps is calculated as an integer part of the product $N_{t}=[T \times 10]$ and $\tau=T / N_{t}$. This procedure confines $\tau$ between 0.1 day and $0.1+0.1 / N_{t}$. The periodic orbits are supposed to be composed of a number of time steps, so the addition of $0.1 / N_{t}$ is small and does not violate the stability.

But $N_{t}$ is an integer and its variations may result in discontinuities of the functional $J$. The jumps of $J$ may occur at any change of $N_{t} \rightarrow N_{t} \pm 1$. These jumps are rclatively small, thanks to the convergence of the scheme, however they can disturb the iterational descent procedure when $J$ becomes small in the vicinity of its zero. One example of this situation is illustrated in fig. 2 .

However, it is easy to avoid the difficulties related to these discontinuities. One just needs to verify at each iteration that the integration time is close to the point of change of $N_{t}$. If, in the descent direction, $N_{t}$ should be changed, the procedure must make this step even if the value of functional increases. Thus the descent process jumps over the discontinuities, and can be continued.

Another numerical aspect we can cmphasise is the construction of the adjoint model. As it is known, the Finite Difference (FD) technique leads to the difference between FD of the adjoint model and the adjoint of the FD model (Sirkes and Tziperman, 1997). The first is accurate only within $O(\delta x, \delta t)$ while the second may contain strong computational modes. FE technique of the space discretisation allows us to avoid this difference and to construct an accurate adjoint model. The use of finite differences in time stepping causes the appearance of a computational mode: two-tine-step leap-frog mode in the present case. This mode is rather strong in the beginning of the adjoint model integration, however it vanishes after some integration time and one can hardly find any trace of the computational mode at its final point.

This is illustrated in fig. 3 , by evolutions of the enstrophy of the solution of the tangent linear model (solid line) and of the adjoint model (dashed line). Fragments from the beginning and the end of the adjoint model integration are enlarged to show the computational mode frarly. One wan see strong leap-frog mode at the begin-

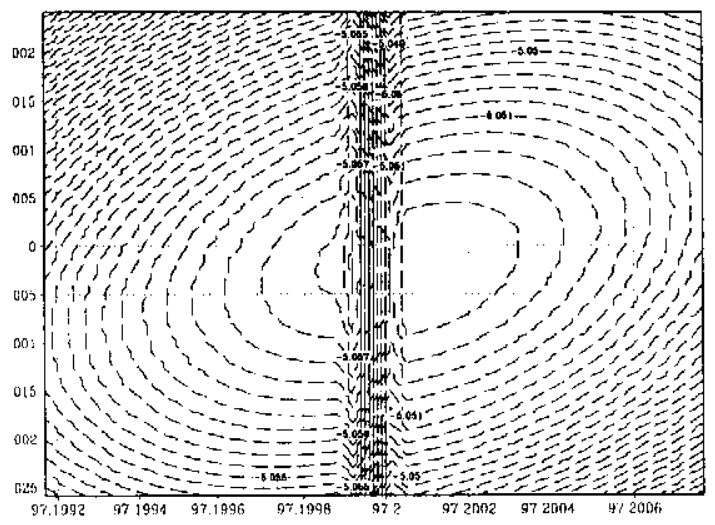

Fig. 2. Logarithm of the functional $J(\xi+h \nabla \cdot J, T)$ as a function of $T$ and $h$. Discontinuity occurs at $T=97.2$ days with the jump $\Delta \ln J \sim 6 \times 10^{-3}$. Contours from -5.057 to -5.046 . Contour interval $2 \times 10^{-4}$

ning of the adjoint model integration (right fragment), although no oscillation can be found at the end of the integration (left fragment).

\subsection{Comparison of different methods.}

Successful development of the descent procedure depends on the matrix used in the scalar product (12) If $\mathcal{H}$ in (12) is equal to the mass matrix, the scalar product becomes an approximation of the $L_{2}$ scalar product. If we chose $\mathcal{H}=I$, we work with a simple Euclidian product in the $R^{N}$ space. In fact, the choice of this matrix results in different preconditioning. The use of the $L_{2}$ scalar product stipulates the equal weighting of all basin regions, while the $R^{N}$ product causes the regions with the refincd grid to weigh more heavily. It is not obvious which scalar product to choose. They are equivalent in general, but this different weighting may accelerate the convergence of the descent procedure. Thus, if the principal variability of the orbit we look for is concentrated in the "refined region" near the jet stream, it is better to use simple $R^{N}$ scalar product which emphasises this region. On the other hand, if the variability of the solution is distributed over the total basin, one should choose the $L_{2}$ scalar product.

Along with approximation of the $L_{2}$ scalar product, we can use the approximation in the $H^{-1}$ space, i.e. the energy of the solution is considered as its norm instead of the enstrophy.

$$
\ll \omega, \varphi\rangle=\iint_{\Omega 2} \Delta^{-1} \omega \varphi d x d y .
$$

In this case the matrix $\mathcal{H}$ must be chosen as

$$
\mathcal{H}=M C^{-1} \mathcal{M} .
$$

To compare the minimisation procedure with now conmon Newton-type methods a referenes search has been 
Table 1. Number of iterations and CPU time necessary for different descent procedures.

\begin{tabular}{|c|c|c|c|c|c|}
\hline Method & $\begin{array}{c}\mathcal{H}=\mathcal{M} \\
\text { (enstrophy) }\end{array}$ & $\begin{array}{c}\mathcal{H}=\mathcal{M C}^{-1} \mathcal{M} \\
\text { (energy) }\end{array}$ & $\begin{array}{c}\mathcal{H}=I \\
\left(R^{N}\right)\end{array}$ & $\begin{array}{c}\text { Newton } \\
\text { method }\end{array}$ & $\begin{array}{c}\text { l)amped } \\
\text { Newton }\end{array}$ \\
\hline Number of iterations & $>2000$ & 910 & 737 & diverges & 69 \\
CPU time & $>115$ & 51.4 & 41.6 & - & 465 \\
Operations per itcration & $O\left(N^{3 / 2}\right)$ & $O\left(N^{3 / 2}\right)$ & $O\left(N^{3 / 2}\right)$ & $O\left(N^{3}\right)$ & $O\left(N^{3}\right)$ \\
\hline
\end{tabular}

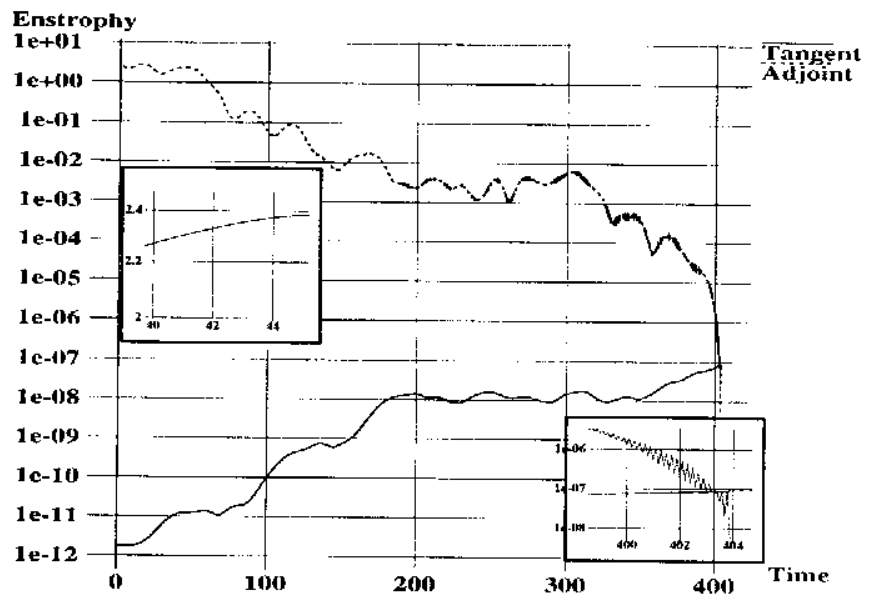

Fig. 3. Evolution of enstrophy of the solution of the tangent linear model (solid line) and of the adjoint model (dashed line). Enlarged fragments from the beginning and the end of the adjoint model integration.

performed beginning with the same initial conditions. The initial state was chosen as an arbitrary state situated not far from the periodic orbit with $T=120.034$. Initial $T$ was taken as $T_{0}=112.8$ with the value of functional $J_{0}=98$.

Five methods have been compared in this search. The first three methods are realisations of the procedure described above. The use of the $L_{2}$ scalar product ( $\mathcal{H}=\mathcal{M}$ ) in the functional definition results in a very slow convergence of the algorithm. In this experiment, the descent procedure does not converge after 2000 iterations. This becomes clear if we note that the variability of the orbit is concentrated in a very small region near the jet stream. This is in agreement also with the result obtained for the variational data assimilation by Luong (1995) who shows that the cnstrophy is not a good norm for the data assimilation procedure.

Following (Luong, 1995), one can hope for more rapid convergence with the approximation of the $H^{-1}$ scalar product, $\left(\mathcal{H}=\mathcal{M C}^{-1} \mathcal{M}\right)$ and this takes place. The process converges after 910 estimations of the functional in 51.4 minutes of CPU time.

Howcver, there is another way to get more rapid convergence. As has been mentioned, the grid refinement near the Western boundary allows us to emphasise the region neatr the jet strearn. In the third run Euclidian product in $R^{N}$ space $(\mathcal{H}=I)$ was used. Minimisational procedure in this example requires 737 estimation of the value of $J$ and its gradient and the CPU time spent for this is 41.6 minutes.

However, we can not definitely state that the difference between scalar products is significant. 1t is just in this experiment that the simple Euclidian norm corresponds better to the minimisation. Sorre other configuration of the periodic orbit in search may make another scalar product more advantageous.

The fourth experiment was performed by the classical Newton method. As has been mentioned, this method diverges rapidly from the initial state. The fifth run uses the dumped-Newton method proposed by Zoldi and Greenside (1997a). This method uses a fraction $\alpha \leq 1$ of a Newton correction $x \rightarrow x+\alpha \delta x$. The value of alpta was taken to be $\alpha=0.02$ if $J>10, \alpha=0.2$ if $1<J<$ 10 and $\alpha=1$ if $J<1$. Of course, the damped-Newtom method converges with the lowest number of itcrations. Only 69 iterations are necessary to reduce the functional value up to the $10^{-20}$. But these 69 iterations require 465 minutes of CPU time, that is more than 10 times the minimisation procedure.

The comparison of the convergence of these five iterational processes is presented in the table 1 .

\section{Periodic orbits, predictability and attractor.}

The idea to approximate the chaotic attractor properties by means of unstable periodic orbits is not new. The important role played by periodic orbits was noted already by H. Poincaré (1892) and E. Hopf (1942). This interest reappears in modern studies. The possibility of studying the strange attractors of dynamical systems by means of periodic orbits is discussed in (Auerbach et al., 1987). Unstable periodic orbits have been found for higher dimensional systems (Kuramoto-Sivashinsky equation with $\mathrm{N}=100$ ) (Zoldi and Greenside, 1997a). The first steps have been performed to distinguish periodic orbits in geophysical systems by Jiang ot al. (1995). Wang and Fang (1996), Itoh and Kimoto (1996), Jiang and Ghil (1997).

In this paper, we try to use low-period orbits to approximate such simple attractor characteristics as its dimension, average values of some solution parameters and their probability clensity functions (PDF).

\subsection{Periodic orbits examples.}

To get an idea of the attractor of the model, we first perform its long-time integration. The mean strommfinc- 

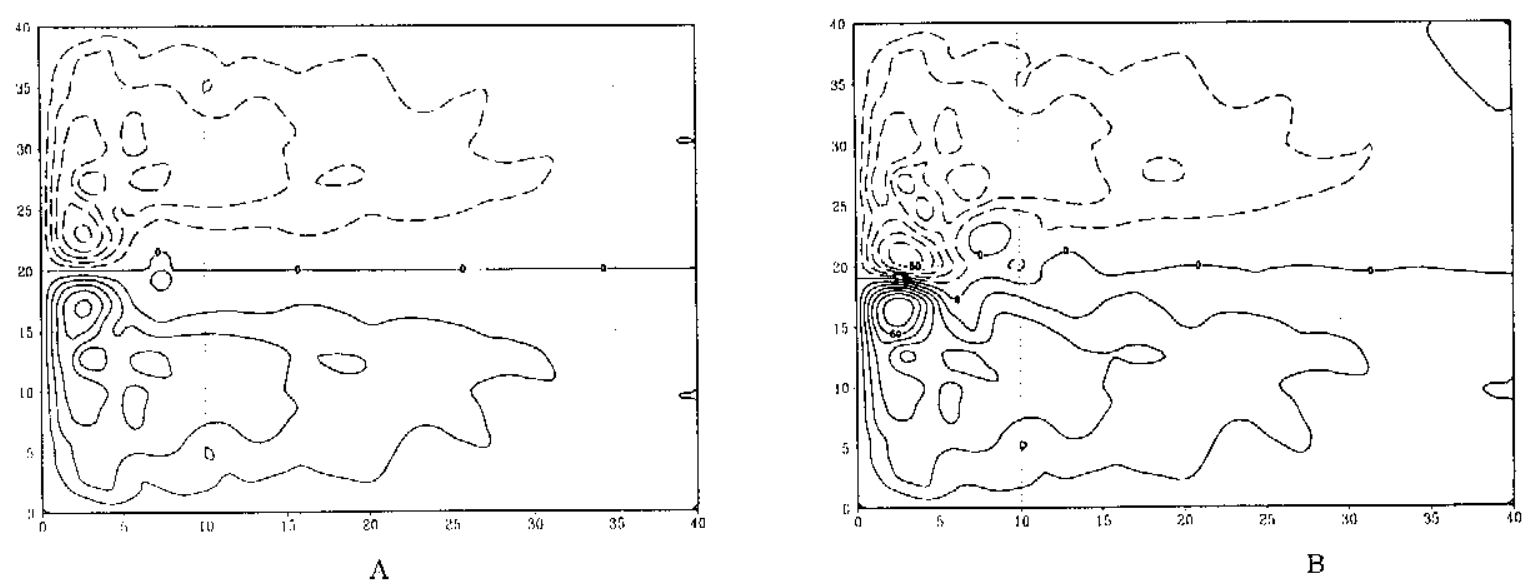

Fig. 4. 5000 years (A) and 50 years (B) averaged streamfunction. Contours from -50 to 50 Sv. Contour interval $10 \mathrm{~Sv}$

tion pattern obtained in this run is presented in fig. $4 \mathrm{~A}$. As one can easily see, the pattern is antisymmetric with respect to the middle line of the basin $(y=2000 \mathrm{~km})$. This symmetry in the long-time average is not surprising. The barotropic model subjected to symmetrical forcing in a square possesses an obvious symmetry; the transformation $\psi(x, y) \rightarrow-\psi(x,-y)$ does not change the system. So its global attractor must possess this symmetry; for any point $\xi(x, y)$ on the attractor, the point $-\xi(x,-y)$ also bclongs to the attractor. Hence, if the system is ergodic, the attractor average must form an anti-symmetrical pattern as we can see in fig. $4 \mathrm{~A}$.

However, only a long-time averaged solution is antisymmetric. For the above model parameters 5000 years of integration is necessary to get an antisymmetric pattern. A typical short-time model run provides a nonsymmetric pattern with the jet-stream directed slightly to the South or to the North. An example of 50 years averaged streamfunction is presented in fig. $4 \mathrm{~B}$. One can easily sce the non symmetry of the jet, which is directed to the South-East. The principal model variability associated with the jet meandering is concentrated bclow the middle line of the domain.

Obviously, the set of states with the jet directed to the North-East and the variability concentrated above the middle line belongs to the attractor also. In fact, the attractor is composed of two basins of attraction. The model spends its time either in one basin with the jet dirccted to the South-East, or in another one, with the jet directed to the North-East.

The model is in the chaotic régime. This fact is illustrated by the energy spectrum of the solution in fig. 5 . Spectral density decreases with frequency. To distinguish principal modes of the variability in this system, we perform the Empirical Orthogonal Functions (EOF) analysis (see Preisendorfer (1988) for a full technical description). We look for an orthogonal basis in the phase space of the model. Each vector of this basis points out the largest variance of the model solution in the subspace orthogonal to the previously found vectors. This

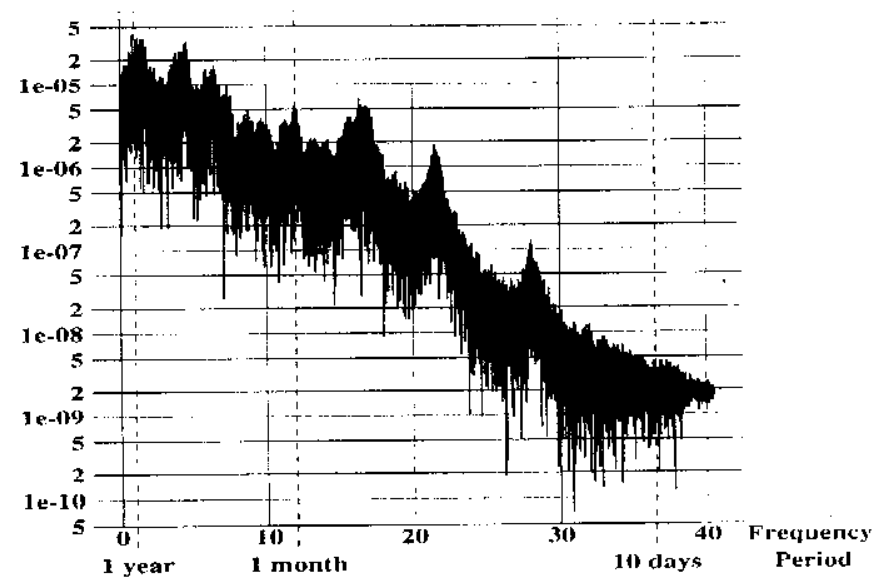

Fig. 5. Energy spectrum of the model solution. Frequency is expressed in year ${ }^{-1}$.

basis can be found as the eigen basis of the covariance matrix.

$C \phi_{n}=\lambda_{n} \phi_{n}, \quad C_{i, j}=\frac{1}{T} \int_{0}^{T}(\mathcal{M} \psi)_{i} \psi_{j} d t$

Eigenvalues $\lambda_{n}$ show the variance associated with cach vector $\phi_{n}$. We arrange the sequence of eigenvectors, or EOFs, so that their corresponding eigenvalues decrease. EOF vectors have been calculated from a 5000 year model run. This integration provides the first EOF symmetric with respect to the middle line of the domain.

The projection of the model solution on the first EOF vector illustrates transitions between basins of attraction with different directions of the central jet. In fact, if the jet is directed to the North-East then the projection on the first $\mathrm{EOF}$ is positive, otherwise the projection is negative. This is caused by the symmetry of the first EOF with respect to the middle line.

One can see in fig. 6 that transitions between these two basins occur irregularly. The lifetime of the model solution in each basin may vary from several dozens to several hundreds years. These rare transitions break the 


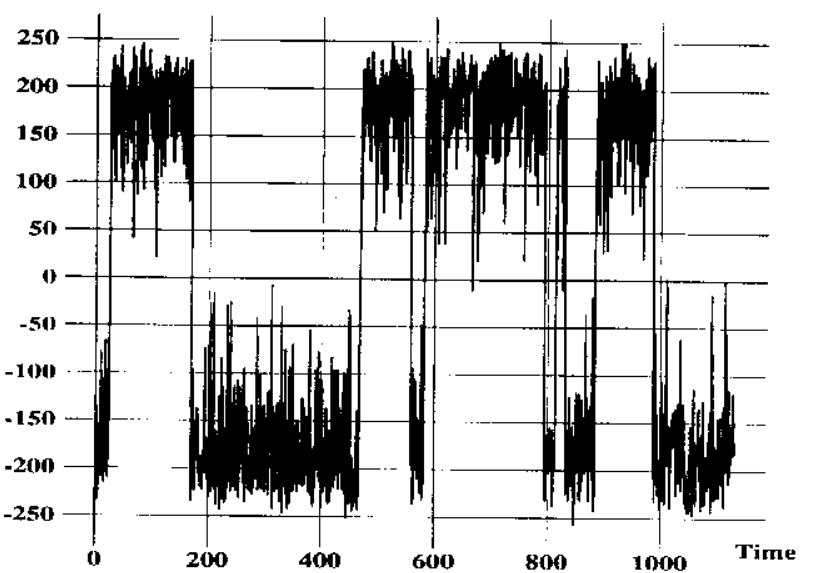

Fig. 6. Time series of the projection of the model trajectory on its first EOF. The time is in years.

symmetry of short time averages of the model solution. Several thousand years integration is necessary to get a number of transitions betwcen these basins and an antisymmetric model average.

This fact provides bimodal PDF of the projection of solution on the first EOF (fig.14A) with two clear maxima. One of these maxima corresponds to the negative projection on the EOF, or to the South-Eastern direction of the jet and the other occurs at positive projection or the North-East jet direction.

This fact has been explained in Dymnikov et al. (1998) by the sequence of bifurcations during the transition of the model to chaos due to increasing the forcing amplitude. However, the evidence of this bimodality can be seen from the periodic orbits configuration.

Three types of orbits have been distinguished on the model attractor from the point of view of symmetry of their averages. The first type includes orbits with the streamfunction antisymmetric with respect to the middle line at any time. Obviously, their average is also antisymmetric. Orbits of the second type allow the jet to be directed both to the South and to the North, resulting in the antisymmetric average. Orbits of the third type are not symmetric. It can be noted here, that the transformation $\xi(x, y) \rightarrow-\xi(x,-y)$ does not change orloits of first two types. Any point on the orbit of the first type is antisymmetric, so $\xi(x, y)=-\xi(x,-y)$. The transformation of an orbit of the second type results in the same orbit shifted one-half period: $S\left(\xi(x, y), \frac{T}{2}\right)=$ $-\xi(x,-y)$. Orbits of the third type are not conserved in this transformation. The transformation applied to any non-symmetric orbits produces the distinct periodic orbit with the same period.

Two orbits of the first type with periods $T=38.14$ and $T=76.6$ days are found. One of them $(T=76.6$ (days) is chosen as an example. Its period averaged strearnfunction is presented in fig.7A. The "y-t," diagram of the evolution of the strennfunction values at $x=500 \mathrm{~km}$ (fig.7B) shows that the solution is always antisymmetric.

One orbit of the second type is found. Its period is $T=41.33$ day. One can see, the period averaged streamfunction of this orbit is antisymmetric (fig. $8 \mathrm{~A}$ ). However, the " $y-t$ " diagram of the streamfunction at $x=500 \mathrm{~km}$ (fig. $8 \mathrm{~B}$ ) shows that the streamfunction is not always antisymmetric. The " $\mathrm{x}$ - $\mathrm{t}$ " diagram of the same orbit illustrates the variability of the streamfunc:tion on the middle line, $y=2000 \mathrm{~km}$ in fig. $10 \mathrm{~A}$.

28 pairs of orbits of the third type are found with periods from 85 to 500 days. One example (orbit with $T=240.5$ days) is shown in fig. 9 . Neither period averaged streamfunction (fig.9A), nor its " $y$-t" diagram are antisymmetric. Complex variability of the streamfunction on the middle line $(y=2000 \mathrm{~km})$ is shown in fig. 10B.

Trajectories of the last two examples are plotted in three-dimensional space of the first 3 EOFs (fig.11). One can sec that the projection on the first EOF always remains positive for the non-symmetric orbit $T=240.5$ (fig.11B) while it changes sign for the orbit $T=41.3$ (fig.11A)

In addition to this, four stationary solutions were found on the attractor of the model. Onc of them is perfectly antisymmetric with respect to the iniddle line, the other three possess no symmetry.

\subsection{Reconstruction of the attractor properties.}

Instability characteristics of periodic orbits are essential quantities, which help to approximate the attractor properties. In this paper, we shall evaluate the instability of orbits in order to verify two hypotheses. The first hypothesis related to the appearance of intermittent oscillations of the model solution and their predictability. The second consists of an approximation to attractor parameters such as dimension and PDF of model solution functionals.

To measure the instability of a periodic orbit we use the sum of positive Lyapunov exponents as a characteristic of the divergence rate of nearby trajectories close to the periodic one. They are based on the eigenvalues of the opcrator of the tangent linear model (14) linearined around the periodic: orbit.

Lyapunov exponents can be calculated as the limit

$\mu_{i}=\lim _{t \rightarrow \infty} \frac{1}{2 t} \ln \lambda_{i}\left(G_{i}^{*} \times G_{l}\right)$ where $G_{t}=\prod_{i=0}^{t} A(S(\xi, t))(29)$

where $A(\xi, t)$ denotes the time step matrices of (16) anst (18) and $\lambda_{i}(G)$ are eigenvalues of $G$. The linit, in the definition of the Lyapunov exponents causes difficultics in their calculation due to the necessity of porforming a very long time integration. However, the use of perioclic orbits can simplify this problem becantse, for any of them, this limit can be calculates in finite time thanks to periodicity. So far, as we know the same orbit, will 


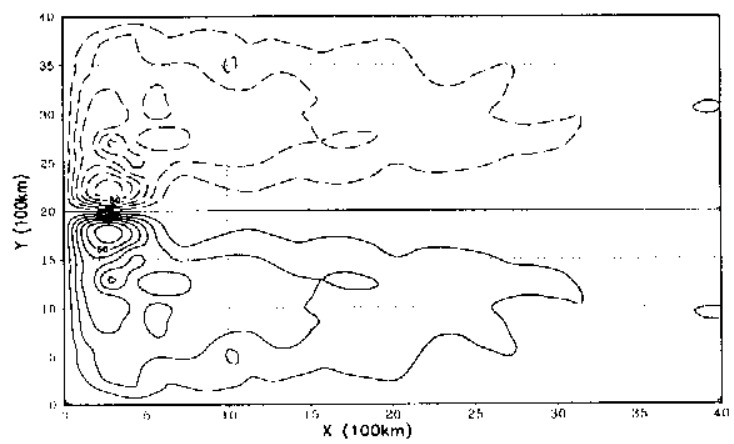

A

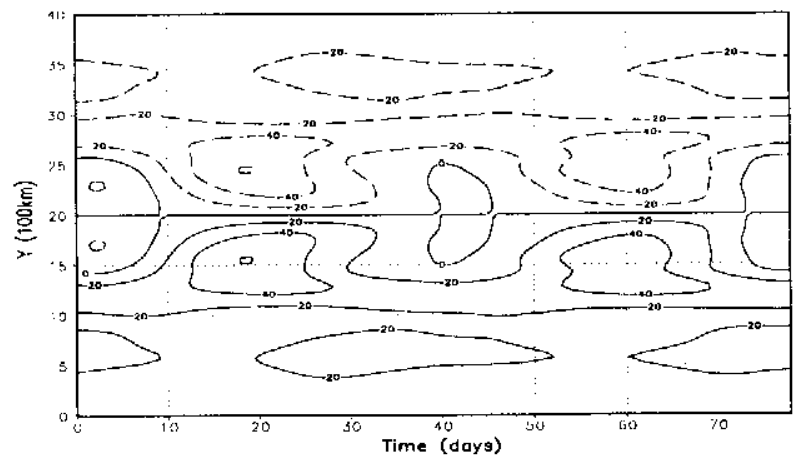

B

Fig. 7. Example of an orbit of the first type (always antisymmetric orbit). Orbit with period $T=76.6$ days. Period-average streamfunction (A) and " $\mathrm{y}$-t," diagram of the streamfunction at $x=500 \mathrm{~km}$ during one period (B).

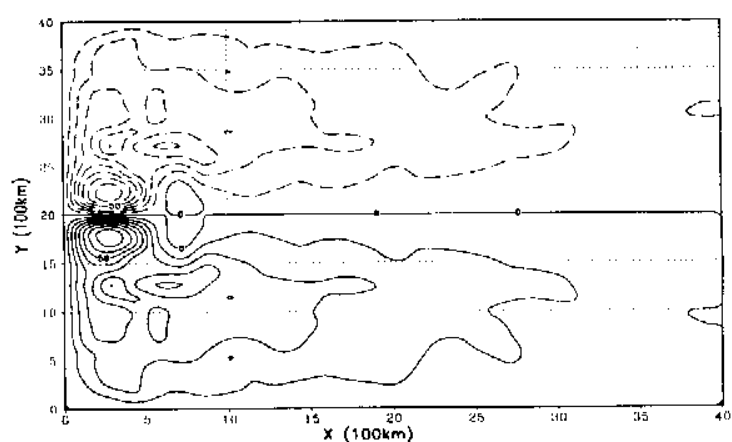

$\Lambda$

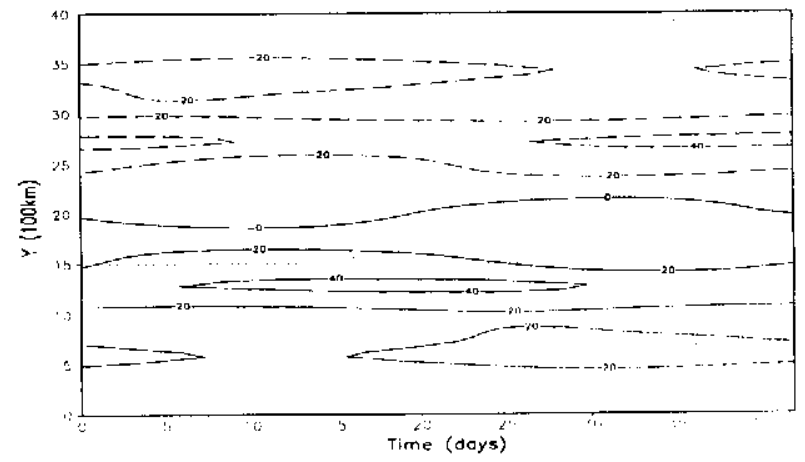

B

Fig. 8. Fxample of an orbit of the second type (orbit with antisymmetric average). Orbit with period $T=41.3$ days. Period-average streamfunction $(\Lambda)$ and " $y$-t" diagram of the streamfunction at $x=500 \mathrm{~km}$ during one period (B).

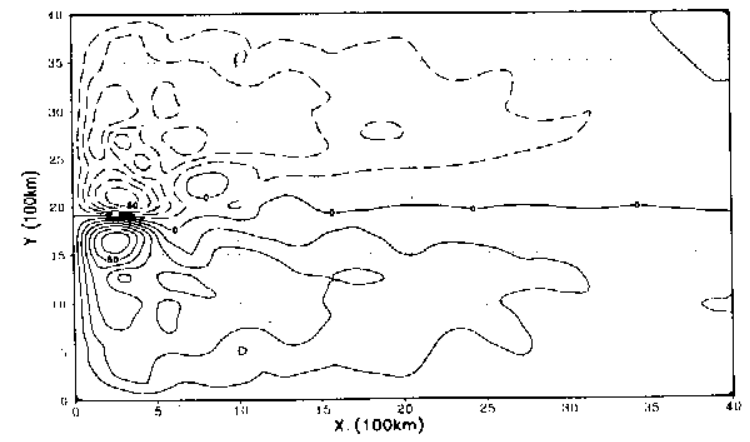

A

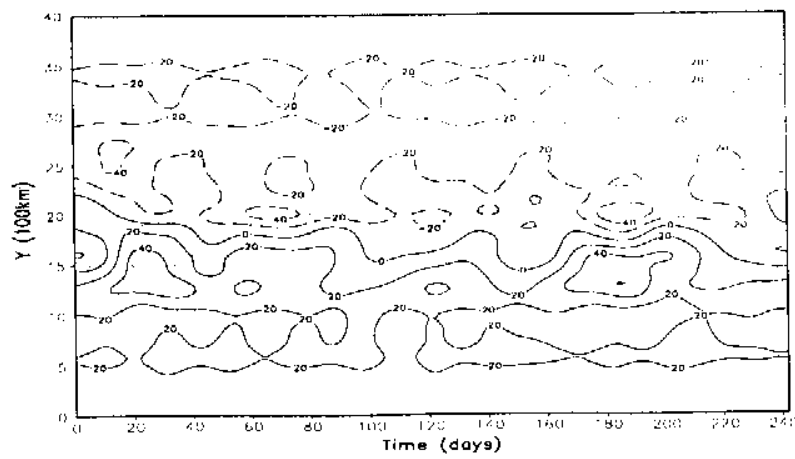

13

Fig. 9. Example of an orbit of the third type (asymmetric orbit). Orbit with period $T=240.5$ days. Period-average streanfunclion $(A)$ and " $y$-t" diagram of the streamfunction at $x=500 \mathrm{~km}$ during one period (B). 


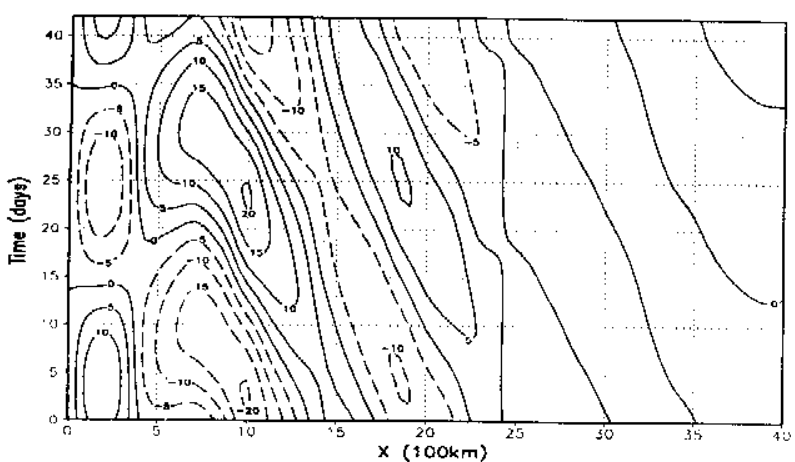

A

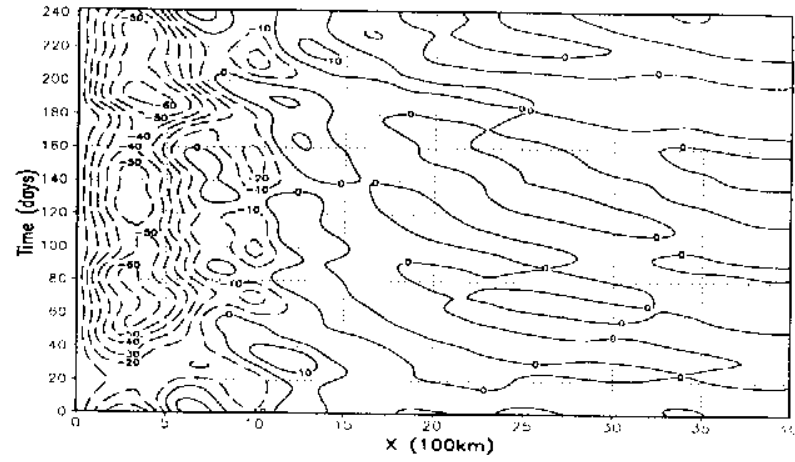

B

Fig. 10. "x-t" diagrams of orbits with $\%=41.3$ days (A) and $T=240.5$ days (B) during one period. Streamfunction is $1.22 k n$ at. $y=2000 \mathrm{~km}$ (middle line)

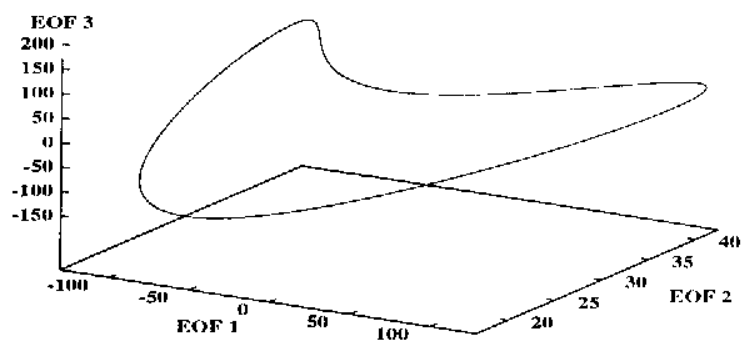

A

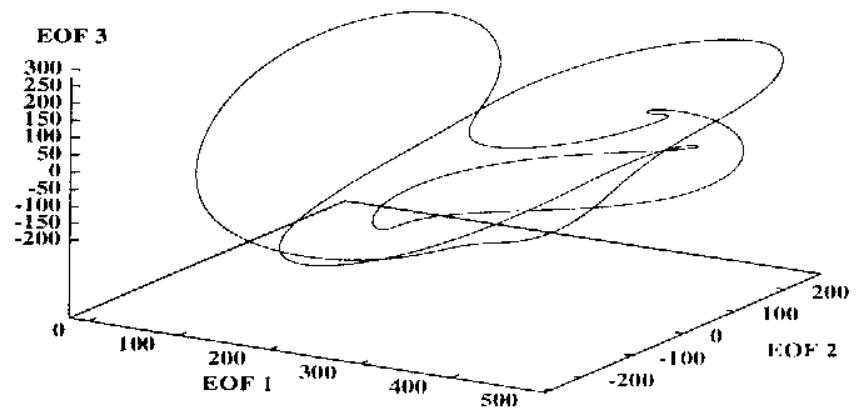

B

Fig. 11. Orbits $T=41.3$ days (A) and $T=240.5$ days (B) in the space of 3 EOF's.

be repeated all the time, we replace the limit $\lim _{t \rightarrow \infty}$ by $\lim _{n \rightarrow \infty}$, where $n$ is the number of repctition of the orbit. So

$$
\begin{aligned}
\mu_{i} & =\lim _{n \rightarrow \infty} \frac{1}{2 n T} \ln \lambda_{i}\left(\left(G_{T}^{*}\right)^{n} \times\left(G_{T}\right)^{n}\right)= \\
& =\frac{1}{T} \ln \left|\lambda_{i}\left(G_{T}\right)\right|
\end{aligned}
$$

Thus, the Lyapunov exponents are related to the Floquet multipliers and can be calculated within one period integration. These Lyapunov exponents can be used to calculate the local attractor dimension by the Kaplan -Yorke formulit.

$\operatorname{Dim}_{\text {local }}=J+\frac{\sum_{i=1}^{J} \mu_{i}}{\left|\mu_{J+1}\right|}$,

where $J: \sum_{i=1}^{J} \mu_{i} \geq 0$, but $\sum_{i=1}^{J+1} \mu_{i}<0$

As it was mentioned in the introduction, the study of the stationary points of the system of the barotropic atmosphere and the corresponding quasi-stationary régimes of atmospheric circulation brought fruitful results in the domain of analysis and a priori estimates of the lifetime of such régimes.
These estimates are based on the supposition that a quasi-stationary régime arises when the trajectory approaches an unstable equilibrium through its stable manifold and withdraws from it through the unstable one. It is natural to deduce that the mean duration of the trajectory's stay in the vicinity of an equilibrium is proportional to the charasteristics of the amstable manifold of the equilibrium. Hence the mean deration of the dis. culation régime becomes proportional tos the instubility characteristics.

In this model study we try to develop this idea and apply it to the periodic solutions, and consequently to define quasi-oscillatory régimes. The existence of such régimes is a well studied phenomenon in physical systoms like the atmosphere or ant ocean. However, the question is open whether they can be explained by the presence of an unstable periodic orbit nearby.

Wo shall use the hypothesis that a quasi-oseillatory régine arises whon the trajectory approaches an mnstirble periodic orbit. Similar to equilibria studies, the tine? spent in the vicinity of a periodic orbit is supposed to be inversely proportional to the instability characteristics of the periodic orbit. The instability characteristic: used here is the sum of positive Lyapunov exponents of the orbit. This value was chosen following the predictability studies of stationary points. However, when 
the closed orbit is considered instead of an equilibrium, it is reasonable also to suppose that the time spent in the vicinity is directly proportional to the volume of the vicinity, i.e. to its length, or its period.

In order to verify this hypothesis, the fraction of time spent by the trajectory in the vicinity is estimated directly. We define $\varepsilon$-vicinity of the periodic orbit as a torus centred on the orbit with circular section of radius $\varepsilon$. Any penetration of the trajectory into a periodic orbit's vicinity during 5000 years integration is registered and the time the trajectory spent in the vicinity is measured. The fraction of time is obtained as the relation of the time spent in the vicinity to the total integration time.

By definition, Lyapunov exponents represent the divergence rate of infinitesimal perturbation. Therefore, the relationship between them and the time spent in the vicinity is not straightforward due to finiteness of real vicinity and due to possible non-symmetry of the operator $G$ which leads to the "super-exponential error growth" (Nicolis et al., 1995). However, the Lyapunov exponents remain good estimates of the fraction of time spent in finite vicinity for the barotropic ocean model.

The choice of radius of the vicinity is somewhat ambiguous. If the vicinity is too large, linear theory on which the Lyapunov exponents are based may fall to approximate non-linear dynamics. On the other hand, a very long time integration is required to get a statistically significant estimate of the fraction of time spent in a small vicinity. In this paper, the radius of the vicirity was taken as 0.04 , that is approximately $10^{-3}$ of the attractor characteristic diameter.

In fig.12, one can see the relationship between the fraction of time spent in the vicinity of the periodic orbit and the value $w_{i}$ equal to the period of the orbit divided by sum of its positive Lyapunov exponents. One can see that the correlation between these values remains high $(0.93 \pm 0.05)$ for the vicinity as large as 0.04 .

Another attractor characteristic, that we can approximate with periodic orbits, is the dimension. The fractal dimension has been calculated directly for this model in Dymnikov et al. (1998) by the Kaplan-Yorke formula and found to be equal to $D=5.8 \pm 0.2$. In this paper, we approximate this dimension by weighted averages of local attractor dimensions of particular orbits.

Zoldi and Greenside (1997a) have suggested using weights equal to the inverses sums of positive Lyapunov exponents. The reasoning of this is clear; less unstable orbits must be weighted more heavily. So the attractor dimension is approximated as

$$
\operatorname{Dim}_{n \text { pprox }}^{(N)}=\frac{\sum_{i}^{N} w_{i} \operatorname{Dim}_{P O_{i}}}{\sum_{i}^{N} w_{i}}, \quad w_{i}=\frac{1}{\sum_{j} \lambda_{i, j}^{+}}
$$

However, it is reasonable also to suppose that orbits with longer periods must be weighted more heavily as they are longer and should provide a greater contribution to

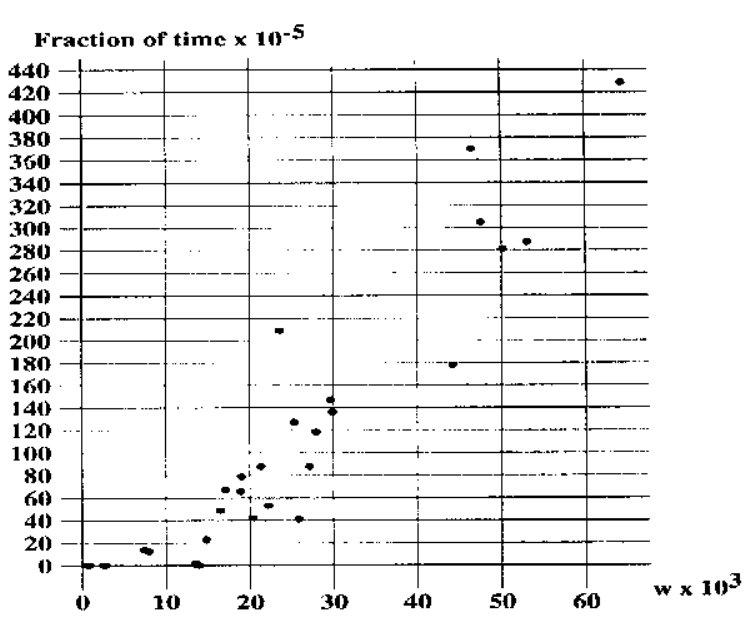

Fig. 12. Fraction of time spent by a trajectory in the vicinity of the PO vs its weight $w$ used in (34).

the total sum. Thus, the weights in this paper are chosen to be proportional to the period of the orbit and inversely proportional to its sum of positive Lyapunov exponents:

$\operatorname{Dim}_{\text {approx }}^{(N)}=\frac{\sum_{i}^{N} w_{i} \operatorname{Dim}_{P O_{i}}}{\sum_{i}^{N} w_{i}}, \quad w_{i}=\frac{T_{i}}{\sum_{j} \lambda_{i, j}^{+}}$

The approximated dimension, calculated using a different number of periodic orbits, is presented in fig.13. Local attractor dimensions associated with particular periodic orbits are marked by dots. One can see that they may vary over a wide range from 4.2 to 18 . However, the weighted mean Dimapprox calculated for different numbers $N$ of periodic orbits, converges to the value of 5.8 . In fact, 25 pairs of orbits with period lower than 400 days are sufficient to approximate the global dimension with $5 \%$ accuracy.

Along with the attractor dimension, we can try to approximate the distributions, or the probability density functions (PDF) of some solution parameters on the attractor.

The distributions can be calculated directly from the long orbit. However, a long integration of the model must be performed to obtain sufficient accuracy due to the slow convergence to the limit. To avoid the necessity of the long model integration, we can use periodic orbits to approximate these distributions. This kind of reconstruction of the PDF has been performed for the Lorenz model by Zoldi (1998) as weighted averages of particular distributions of this parameter on periodic orbits. The weights used in Zoldi (1998) are the same as (33). However, we can get better result for the approximation using weights proportional to the period of the orbit:

$\eta_{\text {approx }}^{(N)}=\frac{\sum_{i}^{N} w_{i} \eta_{i}}{\sum_{i}^{N} w_{i}}, \quad w_{i}=\frac{T_{i}}{\sum_{j} \lambda_{i, j}^{+}}$.

where $\eta_{i}$ is the PDF obtained for the ith orbit. 


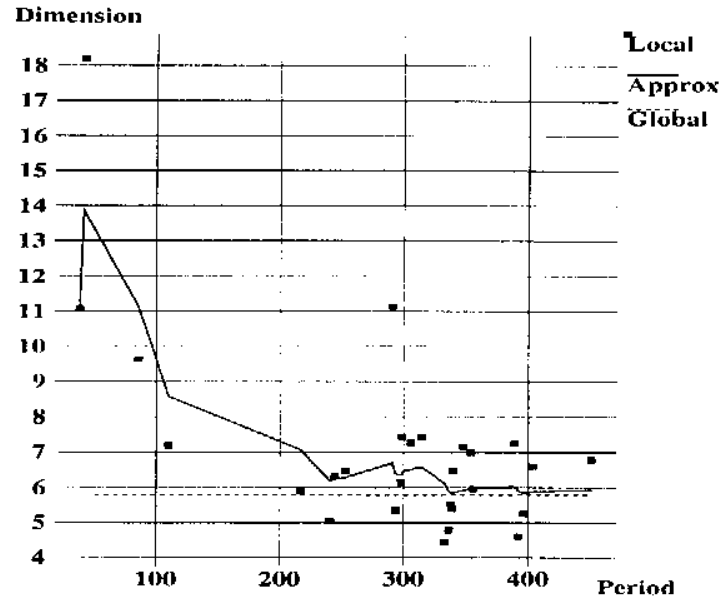

Fig. 13. Local $\Lambda$ ttractor dimension vs period of the orbit (dots). Approximation of global attractor dimension by weighted averages of local attractor dimensions of orbits with periods lower than $\mathrm{T}$ as a function of $\mathrm{T}$ (solid line).

In order to compare the reconstruction of distributions realised by (34) and (35) with the reconstruction proposed by Zoldi and Greenside (1997a), we perform both reconstructions and compare their differences with the value calculated from a long trajectory. This comparison is presented in the table 2 .

One can see that the error obtained when approximations $(34),(35)$, are used is about 2 times lower in all calculations so we use it for the PDF reconstructions.

We compare the PDF of enstrophy and PDF of the projection on the first EOF vector, calculated directly from a long time integration and approximated PDFs outained as a weighted average of PDF's for a limited number of low-period periodic orbits. The direct calculation has been performed for 5000 ycars. This reconstruction was performed using two orbit sets. The first set is composed of 11 lowest period orbits only, i.e. 3 antisymmetric orbits of the first two types and 4 pairs of non symmetric orbits of the third type. The second set includes all 59 found orbits (3 antisymmetric and 28 pairs). The result of comparison is presented in fig.14. We note, that 11 orbits are not sufficient to obtain a good approximation. The difference between the PDF calculated directly and the reconstructed one reaches $35 \%$ due to the presence of numerous spurious maxima and minima in the reconstructed PDF. However, the approximation by 59 orbits provides much a better approximation with an crror of order of $5 \%$.

\section{Conclusion}

The numerical method used to calculate periodic orbits allows us to find some low-period orbits for simple models of the atmosphere and ocean like barotropic or multi-level quasi-geostrophic ones. Implementation of this method requires similar techniques to the data assimilation procedure, which is very well developed for this kind of model.

The knowledge of low-period periodic orbits allows us to approximate some attractor properties which are difficult to calculate directly. As is shown, only a few orbits are sufficient to reconstruct the PDF of the model trajectory or the attractor dimension. The identification of quasi-oscillatory régimes and estimates of their lifetime may be used in predictability studies in a similar manner to the quasi-stationary régimes theory. High correlation between stability measures and the time spent in the vicinity of periodic orbits may allow the use of these orbits and their stability characteristics as an approximation of the invariant measure on the attractor in some cases. In fact, this approximation can be justified only when the periodic orbits set is dense on the attractor of the model.

However, there exist a number of open questions. First. of all, concerning the propcrties of the original model presented as a system of partial differential equations (PDE). Even if we know that an unique solution exists and also an attractor of a PDE system, the cuestion of existence of periodic orbits should be studied carefully. The question of convergence of orbits of the discretised system to orbits of the original PDE system is opern also. Moreover, the density of periodic solutions on the maximal attractor is less evident, even for discretiserl system. The presence of an unstablo insariant torus on the attractor may lead to the fact that orbits are dense on some attractor subsets. Hence it is not evident that. either the whole attractor set can be approximated by orbits, or a subset of the attractor only.

The orbits encoding, application of symbolis: dynamics and possible cycte expansion (sce for example Arutso et al. (1990)) allow us to estimate easily many attractor properties and the predictability characteristics for simple systerns like the Lorenz one (Eckhard and ()t.t. 1994), (Franceschini et al., 1993). In particular cncot. ing of periodic orbits allows ns to know whether all the solutions with periods $T$ less than some $T_{0}$ have hanth found or not. For any missing orbil, we can get a gourl initial guess for the descent procedure and thus find it . Application of the cycle expansion theory allows us to approximate missing orbits with higher periods and even the total periodic orbits set. However, the symbolic dynamics of orbits of a high dimensional system obtained after discretisation of a PDE system is difficult (or innpossible) to understand.

\section{Acknowledgements}

The author is grateful to Christine Kazantsev for rory helpful discussions and to J.C. Gilbert and C. Lentarechal (INRIA, France) for the opportunity to use the cock n1qn3 of the minimisation procedure. All the contour pictures have been prepared by the Grid Analysis and Display System (GrADS) developed in the Centre for Ocean-Land-Atmosphere Interactions, Department of Meteorology, University of Maryland. This work has been accomplished in frames of the Project No.4 of the French- 
Table 2. Comparison of different weighting in the approximation of model averages.

\begin{tabular}{|l|c|c|c|c|c|}
\hline & $\begin{array}{c}\text { Attractor } \\
\text { dimension }\end{array}$ & $\begin{array}{c}\text { Mean } \\
\text { enstrophy }\end{array}$ & $\begin{array}{c}\text { Dispersion } \\
\text { enstrophy }\end{array}$ & $\begin{array}{c}\text { Mean } \\
\text { EOF }\end{array}$ & $\begin{array}{c}\text { Dispersion } \\
\text { EOF }\end{array}$ \\
\hline Long timc & 5.8 & $1.80 \times 10^{-12}$ & $7.27 \times 10^{-14}$ & -6.19 & 349.5 \\
Approx. by (34), (35) & 5.9 & $1.80 \times 10^{-12}$ & $7.35 \times 10^{-14}$ & -6.19 & 350.8 \\
Approx. hy (33) & 6.0 & $1.80 \times 10^{-12}$ & $7.41 \times 10^{-14}$ & -6.18 & 352.2 \\
Error in (34), (35) & 0.1 & $2 \times 10^{-16}$ & $8 \times 10^{-16}$ & $5 \times 10^{-3}$ & 1.3 \\
Error in (33) & 0.2 & $4 \times 10^{-16}$ & $1.6 \times 10^{-15}$ & $1 \times 10^{-2}$ & 2.7 \\
\hline
\end{tabular}

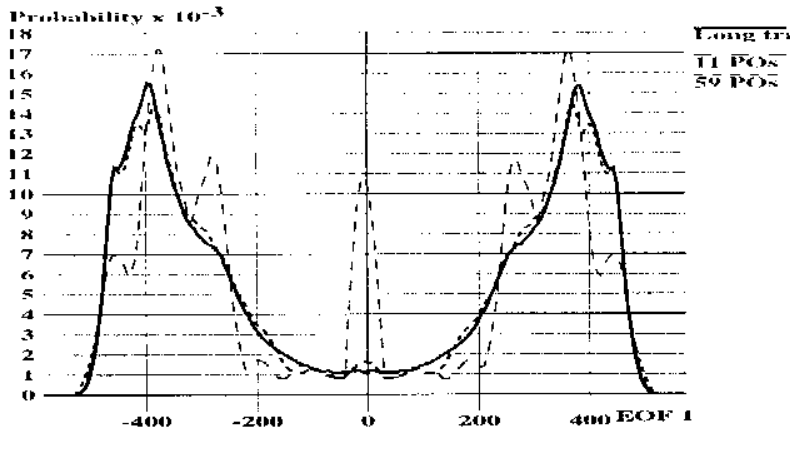

A

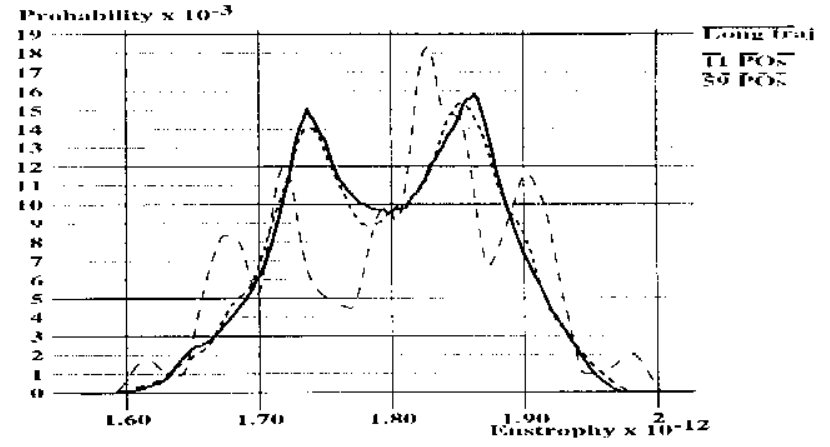

$\mathrm{B}$

Fig. 14. PDF of the projection of the model trajectory on the $1^{\text {th }}$ EOF (A) and PDF of enstrophy (B). PDF calculated directly is drawn by the solid line. Reconstructed PDF with 11 orbits $\left(\eta_{a p p r o x}^{(11)}\right)$ is drawn by a dashed line with long dashes. Reconstructed PDF with 59 orbits $\left(\eta_{\text {tapprox }}^{(59)}\right)$ is drawn by a dashed line with small dashes.

Russian Lyapunov Institute.

\section{References}

Arutso, R., Aurell, E., and Cvitanovic, P., Recycling of strange sets: 1. Cycle expansion., Nonlinearity, 3, 325, 1990 .

Auerbach, D., Cvitanovic, P., Eckmann, J.-P., Gunaratne, G., and Procaccia., I., Exploring chaotic motions through periodic orbits., Physical Review Letlers, 58, 2387, 1987.

Barreto, F., Hunt, B. R., Grebogi, C., and Yorke, J. A., From high dimensional chaos to stable periodic orbits: the structure of paramcter space, Physical Review Letters, 78, 4561-4564, 1997.

Berggren, R., Bolin, B., and Rossby, C., Mn acrological study of zonal motion, its perturbation and break-down., Tellus, $t$, $14-37,1949$.

Bernadou, M., Modulcf: une bibliothèque modulaire d'éléments finis., INRIA, 1988

Bernier, C., Existence of attractor for the quasi-geostrophic approximation of the Navier-Stokes equations and estimate of its dimension., Advances in mathematical sciences \&s Applic., 4, $465-489,1994$.

Blinova, E., Hydrodynamic theory of pressure waves, temperature waves and centres of action of the almosphere., Dokl. AN USSK, 39, 194.3.

Charney, J. and De Vore, J., Multiple flow equilibria in the atmosphere and blocking., Journal of the Atmospheric Sciences, $36,1205-1216,1979$.

Devaney, R., An introduction to chaotis dynarnical systems., Addison-Wesley, 1987.

Dymuikov, V. and Filatov, A., Slability of the large-scale atmospheric processes., Hydrometeoizdat, Leningrad, 1990.
Dymrikov, V. and Filatov, A., Mathematics of Climble Mod elling., Birkhauser, 1996.

Dymnikov, V. and Kazantsev, E. On the attractor structure generated by the system of equation of the barotropic atrmospherr. Izvestiya, Atmospheric and Oceanic Physics, 29, 1993.

I)ymnikov, V., Kazantsev, E., and Kharin., V., Stability measures and lifetimes of atmospheric circulation regimes, Izvesliya, $A t$ mospheric and Oceanio. Physics, 26, 1990.

Dymnikov, V., Kazantsev, C., and Kazantsev, E., On the "genetic. memory" of chaotic altractor of the barotropic ocean model, Chaos, Solitons is Fractals, submitted, 1998.

Eckhard, B. and Ott, G., Periodic orbit analysis of the Lorenz attractor., Zeitschrift für physik. B, 9.3, 259 266, 1994

Filatov, A., Fistimation of the number of instable stationary sointions for spectral rodels of the abmosphere. Works of the Russian Hydro-Metcorological Centre., 1992.

Fix, G., Finitc clements models for ocean circulation problems., J. Appl.Malh., 29, 371, 1975.

Franceschini, V., Giberti, C., and Zheng., Z., Characterisation of the Lorenz attractor by unstable periodic orbits., Nonlinearity, $6,251-258,1993$.

Gilbert, J. and Lemarechal, C., Some numerical experiments with variable storage quasi-Newton algurithrns, Mathematical programming, 45, 407-435, 1989.

Hlunt, B. and Ot, E., Optimal periodic orbits of chantic systens. Physical Review Letters, 76, 2254, I996ia.

Hunt, B. and Ott, W., Optinal periodic orbits of thatic systems occur at low period, Physical Revicu $E, 5,4,329,19966$.

Itoh, II and Kimolo, M. Multiple attractors and clatolic itine ancy in a cuasi-geostrophic mokel with realistic lopongaphy Implications for weather regintes ard low frequenoy variability. Journal of the Atmespheric betences, 5.3. 2217 22.31. 19966.

Jjang, S. and Ghil, M., Tracking nomlimegr solutions with simmlated altimetric data in a shallow-water model, fournal of the 
Physical Oceanography, 27, 72-95, 1997.

Jiang, S., Jin, F., and Ghil, M., Multiple equilibria, periodic and aperiodic solutious in a wind-driven double gyre shallow-water model., Journal of the Physical Oceanography, 25, 764-785, 1995.

Kazanlsev, E., Unstable periodic orbits and attractor of the Lurenz model., Research report 3344, INRIA, http://www.inria.fr//INRIA/publication/publi-psgz/RR/RR-3344.ps.gz, 1998.

Le Provost, C., Bernier, C., and Blayo, E., A comparison of two numerical methods for integrating a quasi-genstrophic multilayer model of ocean circulations: finite clement and finite difference methods., Journal of Computational Physics, 110, 1994.

Legras, B. and Ghil, M., Persistent anomalies, blocking and variations in atmospheric predictability., Journal of the Atmospheric Sciences, 12, 433-471, 1985.

Lorenz, E.., Deterministic non periodic flow., Journal of the Atmospheric Sciences, 20, 130 141, 1963.

Luong, B., Techniques de contrôle optimal pour un modele quasi-gćostrophique de circulation océanique. Afplicalion is l'assimilation variationelle des données altimétriques satellitaires, Ph.D. thesis, Université J. Fourrier, Grenuble, France, 1995.

Mo, K. and Ghil, M., Statistics and dynamics of persistent anomalies, Jourtial of the Atmospheric Sciences, 44, 877-901, 1987.

Mo, K. and Ghil, M., Cluster analysis of multiple planetary flow regimes., Jourral of Geophysical Research, 93, 10927-10952, 1988.

Nicolis, C., Vannitsem, S., and Royer, J.-F., Short-range predictability of the atmosphere: Mechanism for superexponcntial error growth., Quarterly Journal of Royal Meteorological Sicicty, 1\%1. 705-722, 1995.

Preisendorfer, R., Principal Component Analysis in Meteorology and Occanography., Elsevier, 1988.

Rex, T)., Blocking action in the middle tropospherc and its effect on regional climate., Tellus, $2,275-301,1950$.

Rossby, C., Relation between variations in the intensity of the zonal circulation and the displacement of the semi-permanent centers of action, J.Mor. R.es., 2, 38-55, 1939.

Schmelcher, P. and Diakonos, F., A general approach to the localization of unstable periodic orbits in chaotic dynamical systcms, submitted to Physical Review k, I.ANL c-print (ht.t.p://xxx.lanl.gov) chao-dyn/9711007, 1997.

Sirkes, Z. and 'rziperman, E., Finite difference of adjoint or adjoint. of firite difference, Monthly Weather Review, 125; 3373 33.37\%, 1997.

Sparrow, (․, The Lorenz Eifuntions: Bifurcations, Chaos and Sitrantye Altraclers, Springer, 1982.

Tomam, R., Infinite-dimensional dynamical systems in mechanics and physics.s, no. 68 in $\Lambda_{\text {pplied Mathematical Sciences, }}$ Springer-Verlag, I!j88.

Wang, B3. and Fang, Z., Chaotic oscillations of tropnical climalle: A dyuanic system theory for enso, Jonrnal of the Atmospherir: Sciences, 53, 2786-2802, 1946.

Zoldi, S., Unst,able: periodic orbits analysis of histograms of clantic: time series, submilled to Physical hevien Letlers, $1, \triangle \mathrm{Nl}$, (-print. (htt.j)://xxx.liznl.gov) chao-dyn1/9802005, 1998.

\%oldi, S. and Corenside, H., Spatially localized unstable periodic: orbits., submilled to Physical Review Lellers, J.ANI, e-prinl. (lit.tp://xxx.lant.gov) chao-dyro/9704095, J997at.

Zokli, S. and Cirentsicle, Il., Comment on optimal periodic: or-

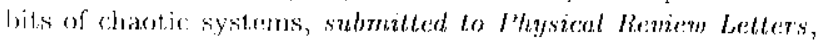
I.AN], (-print. (h1.tp://xxx.lenl.gov) chano-dyn/9707001, 1997b, 